\title{
When Does Immune Recovery Occur After Stem Cell Transplantation?
}

\section{Kök Hücre Nakli Sonrası İmmün Düzelme Ne Zaman Olur?}

\author{
Eda Kepenekli Kadayıfçı ${ }^{1}$ \\ ${ }^{1}$ Division of Pediatric Infectious Diseases, Marmara University Pendik Training and Research Hospital, Istanbul, Turkey
}

Cite this article as: Kepenekli Kadayıfçı E. When does immune recovery occur after stem cell transplantation? J Pediatr Inf 2019;13(3):e97-e102.

Abstract

Hematopoietic stem cell transplantation has been increasingly used in the treatment of many diseases since the 1950s. Post-transplant immune reconstitution is depended to the stem cell source, immuno suppressive interventions used, complications, the cell subgroups which can improve at different times. Immune reconstitution starts right after stem cell transplantation and can extend up to five years.

Keywords: Hematopoietic stem cell transplantation, HSCT, immune reconstitution

Stem cells are the cells that can renew themselves, be induced to transform into different cells, engraft and multiply from a single clone. The idea that these cells can be used in the treatment of some diseases has been accepted after animal experiments. In the 1950s, the basics of stem cell transplantation were laid by demonstrating that irradiated mice can survive with the infusion of spleen and bone marrow cells separated prior to the irradiation (1). Thus, in later periods, stem cell transplantation was started to be used in the treatment of certain benign or malignant diseases, which are unresponsive to conventional therapies. From the years stem cell transplantation was used for the first time until today, "preparative" regimens used to suppress the lymphohematopoietic system, source of the stem cell used, criterions of stem cell donors, treatment and prophylactic approaches for the complications that occur during the post-transplantation period have changed and improved and hence, stem cell treatments have become more widely used.

According to the data of the European Society for Blood and Marrow Transplantion (EBMT), while 1-50 individuals per 10 million population received stem cell transplantation in 1998 in our country, this figure increased to the range of 101-150 in 2013 (2).

In the 1950s when human trials of stem cell transplantation started, this method was primarily used for the treatment of cancer, aplastic anemia and primary immunodeficiencies. Today, it is used as a treatment method in many different diseases. These diseases are summarized in Table 1. 
Table 1. Diseases treatable with hematopoietic stem cell transplantation*

\begin{tabular}{|c|c|}
\hline Malignant diseases & Non-malignant diseases \\
\hline Acute lymphoblastic leukemia & Severe aplastic anemia \\
\hline Acute myeloid leukemia & $\begin{array}{l}\text { Inherited bone marrow failure } \\
\text { syndromes }\end{array}$ \\
\hline Myelodysplastic syndrome & Primary Immunodeficiencies \\
\hline $\begin{array}{l}\text { Juvenile myelomonocytic } \\
\text { leukemia }\end{array}$ & Lymphoid deficiencies \\
\hline Chronic myelgenous leukemia & T-cell activated SCID \\
\hline Hodgkin's lymphoma & X-dependent SCID \\
\hline Non-Hodgkin's lymphoma & $\gamma$-chain defect of IL2RG \\
\hline Neuroblastoma & Autosomal recessive types of SCID \\
\hline \multirow[t]{27}{*}{ Brain tumors } & JAK3 defect \\
\hline & IL7Ra defect \\
\hline & ADA insufficiency \\
\hline & $\begin{array}{l}\text { Purine nucleotide phosphorylase } \\
\text { defect }\end{array}$ \\
\hline & RAG1 or RAG2 defect \\
\hline & Omenn's syndrome \\
\hline & Artemis mutation \\
\hline & DNA ligase IV mutation \\
\hline & CD3 chain mutation \\
\hline & CD45 defect \\
\hline & Zap70 mutation \\
\hline & $\begin{array}{l}\text { T and B cell-concomitantly } \\
\text { activated Ataxia telangiectasia }\end{array}$ \\
\hline & $\begin{array}{l}\text { MHC II (bare lymphocyte } \\
\text { syndrome) }\end{array}$ \\
\hline & B cell-activated \\
\hline & $\begin{array}{l}\text { X-dependent } \\
\text { hypogammaglobulinemia of } \\
\text { infancy }\end{array}$ \\
\hline & X-dependent hyperlgM \\
\hline & Mixed immunodeficiencies \\
\hline & Wiskott-Aldrich syndrome \\
\hline & $\begin{array}{l}\text { Immunodeficiencies affecting myeloid } \\
\text { cells }\end{array}$ \\
\hline & Chronic granulomatous disease \\
\hline & Chediak-Higashi syndrome \\
\hline & Leukocyte adhesion defects \\
\hline & Griscelli syndrome \\
\hline & Hemophagocytic lymphohistiocytosis \\
\hline & $\begin{array}{l}\text { Hemoglobinopathies (Beta- } \\
\text { thalassemia major, sickle-cell anemia) }\end{array}$ \\
\hline & Inherited metabolic diseases \\
\hline & Osteopetrosis \\
\hline \multicolumn{2}{|c|}{$\begin{array}{l}\text { * Adapted from reference no } 3 . \\
\text { SCID: Severe combined immunodeficiency. }\end{array}$} \\
\hline
\end{tabular}

Hematopoietic stem cell can be obtained from three sources:

1. Bone marrow,

2. Peripheral blood,

3. Umbilical cord.

While bone marrow is preferred as the stem cell source in the treatment of non-malignant diseases, peripheral blood is preferred mainly as the stem cell source particularly in leukemia. In comparison to other source types, graft-versus-host disease (GVHD) is observed at a higher rate in peripheral blood stem cell transplantation (3).

Classification regarding stem cell donor type is as follows:

1. Syngeneic: Stem cell transplantation from a twin partner.

2. Autologous: This is the collection and preservation of one's own stem cells before ablation and re-transferring them after chemotherapy and/or radiotherapy. It is typically used in the treatment of solid tumors. GVHD is not observed and engraftment occurs early. The fundamental aim in autologous transplantation is to give chance to hematologic recovery after high-dose therapy.

3. Allogeneic: All transplantations where the stem cell source is not the individual him/herself or the twin partner are regarded in this group. It is divided into two subgroups; related and unrelated donor. There are two widely used terms in this transplantation type: match-sibling-donor (MSD) is used to define the procedure where the stem cell donor is the patient's sibling, and match-unrelated-donor (MUD) is used to define transplantations from unrelated stem cell donors. Human leukocyte antigens (HLA) are used to assess compatibility between stem cell recipient and donor. This antigen cluster possesses 10 different alleles, five from the mother and five from the father $(A, B, C, D R$, DQ ; two each). When $9 / 10$ or $10 / 10$ compatibility from these 10 different alleles is detected between the recipient and donor, it is referred to as 'full compatibility". In cases of mis-matched donor (MMD), namely in cases without full compatibility, A, B, DR compatibility is absolutely sought, and compatibility rate can be as $6 / 10$ or 7/10. In haploidentical transplantations, namely in semi-compatible transplantations, (5-6-7/10), GVHD probability is high. However, this condition is preferable in particularly leukemia to produce graft-versus-tumor response. Graft-versus-tumor effect is an important feature of allogeneic hematopoietic stem cell transplantation (HSCT). 
Stem cell transplantation performed in the treatment of malignancies can be autologous or allogeneic. Allogeneic source is mainly used for malignancies like leukemia involving the bone marrow or myelodysplastic syndrome. Autologous source is essentially preferred in solid tumors and some recurrent or refractory lymphoma types. Allogeneic transplantation is used in the treatment of numerus non-malignant diseases, and these diseases are divided into five basic subgroups: acquired severe aplastic anemia, inherited bone marrow failure syndromes, primary immunodeficiencies, hemoglobinopathies and inherited metabolic diseases (Table 1) (3).

Dictionary meaning of 'engraft' is to incorporate, to plant firmly and to graft. The simple definition of engraftment in HSCT is that the recipient's body accepts the implanted stem cells and starts producing new blood and immune cells afterwards. The fundamental aim in HSCT is to provide engraftment and to place the lymphohematopoietic system of the donor instead of the one of the recipient, and to realize these on the grounds of reduced or vanished tumor effect.

Total nucleated cell (TNC) dose and CD34+ cell dose within the transplanted cells during hematopoietic stem cell transplantation are among crucial factors designating engraftment time and result of HSCT. TNC level is low in stem cells originating from the umbilical cord, and neutrophil engraftment (absolute neutrophil count $>500 / \mathrm{mm}^{3}$ ) takes longer. This duration is 14 days in peripheral blood stem cell transplantation (SCT), 21 days in bone marrow SCT and 30 days in umbilical cord SCT (4). It has been reported in peripheral blood SCT that there is increase in all survival and decrease in relapse with elevation of the TNC dose; however, chronic GVHD has been observed at a higher rate (5). It has been reported thrombocyte engraftment occurs earlier, but all survival rates decrease and the number of relapses increase when $\mathrm{CD} 34+$ cell dose in peripheral blood SCT increased (6).

GVHD, namely the state where the transplanted tissue harms the recipient's tissues to be kept healthy, is one of the major problems in allogeneic HSCT. There are two subtypes including acute GVHD and chronic GVHD. Acute GVHD usually develops in the first 100 days after SCT. It is a condition where donor T cells generate response to polymorphic histocompatibility antigens in the host that is not fully compatible. Chronic GVHD develops after the first 100 days following SCT and exhibits characteristics of autoimmune diseases. It can develop de-novo or it can progress as a continuation of acute GVHD.

The process of stem cell transplantation comprises different stages (Figure 1) (1).

1. Conditioning phase: Numerous different chemotherapy, radiotherapy or immunotherapy methods can be applied individually or in combination. Bone marrow

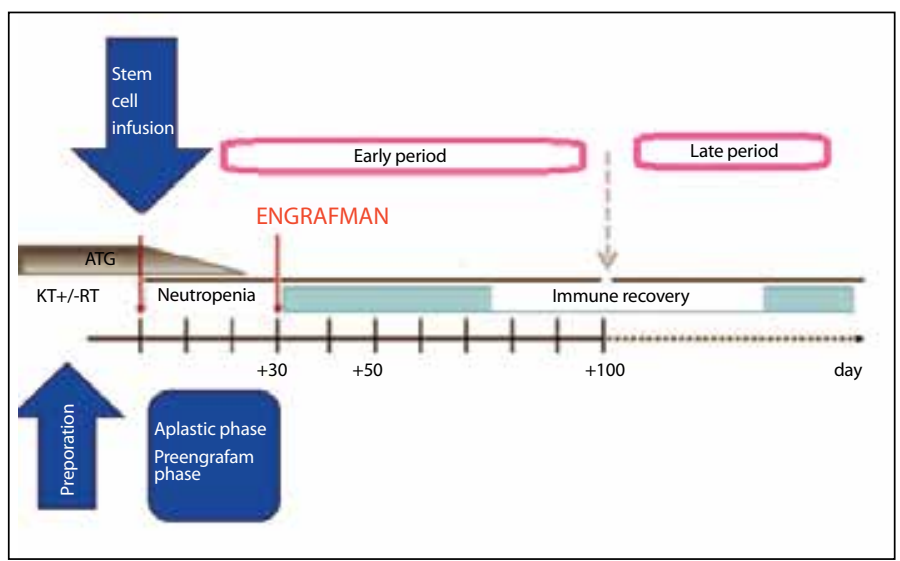

Figure 1. Stages of stem cell transplantation.

can be fully ablated or mild myelosuppression can also be performed. Conditioning regimen can vary according to the stem cell source and GVHD prophylaxis regimen, the characteristics of the patient and the disease and whether there is a donor or not.

2. Stem Cell Infusion:The day of infusion is considered as day 0 .

3. Pre-engraftment period: Also known as the aplastic period. It generally covers the first 30 days after stem cell infusion. The fact that the patient is neutropenic in this period makes him/her more prone to bacterial and fungal diseases. Therefore, the need to use antibiotics and antifungal treatments or prophylaxis arises.

4. Early post-transplantation period (engraftment period): Comprises the first 100 days after transplantation. Cellular immunodeficiency state is dominant and NK cells and T cell count are low. Cytomegalovirus and Epstein-Barr virus infections, reactivations, some other viral infections and Pneumocystis jirovecii infections can be problematic. Acute GVHD can develop at this stage.

5. Late post-transplantation period (post-engraftment period): It is the period that starts after 100 days after transplantation. Viral infections, reactivations and chronic GVHD are features of this period.

In order to better understanding of immune recovery after stem cell transplantation, we can review the differentiation of immune system over Figure 2 (7). In human, differentiation of the immune system starts with hematopoietic pluripotent stem cell. Thereafter, it transforms into the innate immunity and adaptive immunity that are different functionally. While $B$ and $T$ lymphocytes and their subgroups are responsible for adaptive immune response, neutrophils, eosinophils and basophils dendritic cells, monocytes, tissue macrophages and complement system generate innate (natural) immune response. 


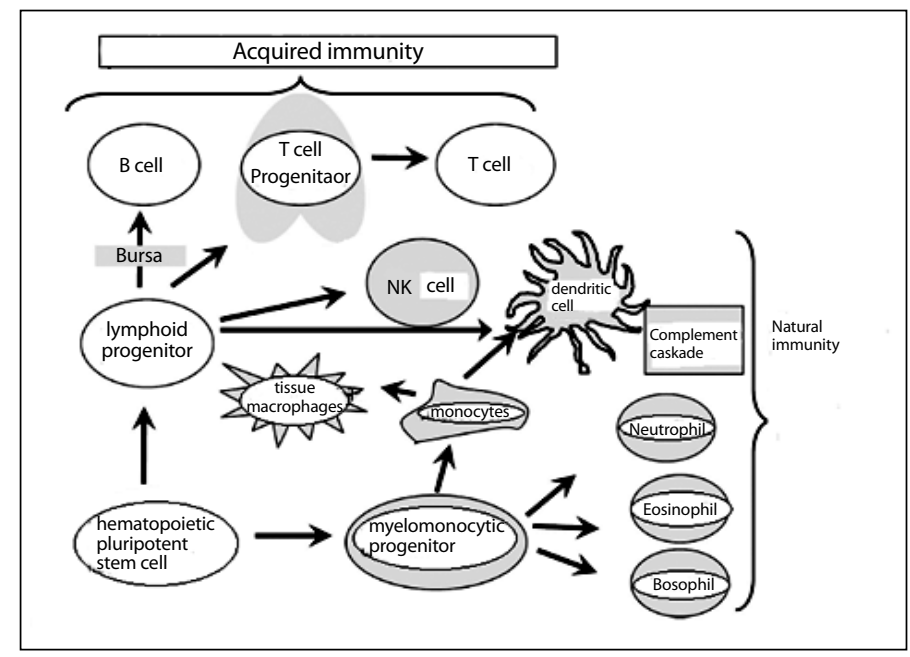

Figure 2. Differentiation of immune system.

Table 2. Immune reconstitution after allogeneic stem cell transplantation*

\begin{tabular}{|l|c|}
\hline Cells & Time span after stem cell transplantation \\
\hline Neutrophil $>0.5109 / \mathrm{L}$ & $\begin{array}{r}\sim \text { days; peripheral blood stem cell } \\
\sim 21 \text { days; bone marrow } \\
\sim 30 \text { days; umbilical cord }\end{array}$ \\
\hline NK cells & $30-100$ days \\
\hline T cells & 100 days \\
\hline CD19+ B cells & $1-2$ years \\
\hline * Adapted from reference no 7.
\end{tabular}

\section{Recovery of Innate Immunity}

Occurs within 20-30 days after hematopoietic stem cell transplantation (Table 2) (7). Respectively, first the monocytes, and then the granulocytes and finally NK cells are restructured (8).

\section{Recovery of Adaptive Immunity}

It can extend to a year after hematopoietic stem cell transplantation. T lymphocytes are expected to gain function again roughly in the first 100 days. The time of lymphocyte function recovery varies with respect to the implemented conditioning regimen, thymic involution of the host, donor age, graft type, stem cell dose, whether or not ex-vivo or in-vivo T cell depletion has been performed, GVHD prophylaxis performed and whether there is GVHD or not. There are two mechanisms for T lymphocytes to gain function:

1. Peripheral survival and expansion of donor T cells,

2. De novo donorT cell production in the thymus from donor hematopoietic precursors.

In the first month of post-transplant period, the mechanism that is in action is the peripheral survival and expansion of donor T cells.
Recovery of $C D 4+$ and $C D 8+T$ cells takes place in the first year after HSCT. Recovery of CD4+ T lymphocytes occurs after CD8+ T lymphocytes and can extend up to 2 years. Generally, $\mathrm{CD} 4+\mathrm{T}$ cells recover within $>100$ days after transplantation. While $C D 4+/ C D 8+$ ratio is in favor of CD8+ $T$ cell during the early periods following transplantation, this ratio becomes reversed with the re-structuring of CD4+ T cell. It is observed that CD4+T cell count is approximately $200 / \mu \mathrm{L}$ about 3 months after HSCT (7).

T lymphocytes are the main directors of viral and fungal infection control and graft-versus-leukemia response. Slow re-structuring of $T$ cell is accepted as the primary responsible of latent viral and fungal infections, graft-versus-host diseases and relapse (7).

Trec (T cell receptor re-arrangement excision DNA circles) are DNA particles that form during the re-structuring of $\mathrm{T}$ cell receptor and are carried in T cells as episome. The presence of this particles in a T lymphocyte shows that cell is 'thymus' based. Therefore, they are used as thymic function marker. $T$ rec levels sustain a low level until $3^{\text {rd }}$ to $6^{\text {th }}$ months after allogeneic HSCT. Low Trec levels have been found associated with the opportunistic infections (9).

RegulatorT Cells (Treg) are a subgroup of CD4+T cells. They suppress effector $T$ cells with the help of inhibitor cytokines including IL-10 and TGF-Beta, and hence, reduce inflammation. Immature T lymphocytes produced in the bone marrow moves to the thymus and then, the lymph node stations and differentiate into regulator $T$ cells that are able to control effector T cells. The balance in the gut microbiome has a critical role in providing tolerance of the regulatory $T$ cells in gut. Regulatory $T$ cells are significant regulators in autoimmunity, acute and chronic infection response, allergies, immune tolerance in pregnancy, tissue damage response, cancer and transplant immunology, and host-parasite interactions (7).

Regulatory $T$ cells ensure immune homeostasis after allogeneic HSCT, and thus play a vital role in prognosis of allogeneic HSCT. T regs are major elements of tolerance both in solid organ transplant and in allogeneic HSCT. They are the most important factors in GVHD recovery. When regulatory $T$ cells have been implanted to the donor graft in animal studies, it has been observed that GVHD reduces and the graft-versus-leukemia effect has been kept (7). Ex-vivo multiplied $C D 4+C D 25+C D 127$ - regulatory $T$ cells were given for the first time to two patients with acute GVHD and chronic GVHD each, and it has been reported that there was an increase in T reg count in circulation, a decrease in inflammatory cytokine levels, and that these patients needed steroids less (10). In a study, it has been emphasized that the elevation inCD4+CD25+ T reg count effects inversely severity of GVHD 
Table 3. Effect of stem cell source on immune recovery and complications*

\begin{tabular}{|l|c|c|c|}
\hline Complication & Peripheral blood SCT & Bone marrow SCT & Umbilical cord SCT \\
\hline Acute GVHD & ++ & + & $+/-$ \\
\hline Infections & + & + & ++ \\
\hline Viral reactivations & ++ & ++ & $+/-$ \\
\hline Relapse & $+/-$ & ++ & ++ \\
\hline $\begin{array}{l}\text { * Adapted from reference no 7. } \\
\text { GVHD: Graft-versus-host disease, SCT: Stem cell transplantation. }\end{array}$ & \\
\hline
\end{tabular}

(11). Currents researchs focus on two questions 1) Can T regs be used in GVHD prophylaxis and treatment? and 2) Can T regs be used to ensure immune reconstitution? Initial findings about these questions seem positive.

Recovery of virus-specific immune response is one of the factors affecting both survival and quality of life after transplantation. $T$ lymphocytes are the most important cells in controlling viral infection. Viral infections are most frequently encountered within the first 90 days after engraftment. The major determinants of reconstitution of virus-specific immune responses are mature (memory) $\mathrm{T}$ lymphocytes of the donor transplanted by the graft, naive T lymphocytes, effector T lymphocytes, and the host's antigen-specific cell count and function (7). In a study investigating cytomegalovirus-specific cytotoxic T lymphocyte response, it has been reported that when both the donor and the recipient were seropositive then virus-specific cytotoxic T lymphocyte count underwent an increase as a response to the cytomegalovirus reactivation detected by pp65 antigenemia, but when the recipient was seropositive and the donor seronegative, virus-specific cytotoxic $T$ lymphocyte response occurred after 100 days despite early cytomegalovirus reactivation (12). Virus-specific T lymphocyte infusion has been tested after allogeneic HSCT and solid organ transplant, and promising results have been obtained.

The slowest part of immune reconstitution takes place in the recovery of $B$ lymphocyte functions. It usually re-gains function in 1-2 years. This period of time can be 6 months in autologous transplantations and 9 months- 5 years in allogeneic transplantations. The fact that different $B$ cell subpopulations enter into the recovery period at different times leads to the formation of a damaged humoral immune response. Delay in the recovery of $\mathrm{T}$ cells and inverse proportion of CD4/CD8 also play a role. The implemented GVHD prophylaxis regimen or GVHD itself contributes (13). Normal IgM levels are started to be observed in 3-6 months after HSCT. Following this, serum $\lg G 1 / \lg G 3, \lg G 2 / \lg G 4$ and $\lg A$ levels ameliorate. Since $\mathrm{CD} 19+\mathrm{CD} 27+$ memory $B$ cells require adjuvant $T$ lymphocytes to conclude isotope transformation, $B$ cell recovery is affected negatively if anti-thymocyte globulin has been given in the conditioning phase. Stem cell source is an important determi- nant in B cell recovery, as well. Much more B cell is determined in recipients of peripheral blood SCT. 'KREC's (kappa-deleting excision circles) are used as markers for the detection of $B$ cell production. The absence of CD19+CD27+ memory B cell, low levels of immunoglobulins, damaged immunoglobulin class transformation, and losses in gene regulation cause predisposition to infections with capsulated bacterias (14).

Table 3 summarizes the effect of stem cell source on immune reconstitution and complications (7). When stem cell grafts with reduced T cell dosage are used, GVHD decreases. However, immune reconstitution is delayed and opportunistic infections and relapses increase. Therefore, there is a fine balance between GVHD development and immune reconstitution. While interventions to decrease GVHD affects immune reconstitution negatively, interventions that improves immune reconstitution can increase GVHD risk.

There are some markers for monitorization of immune reconstitution after HSCT:

1. Absolute lymphocyte count,

2. Lymphocyte sub-group analysis (CD4, CD8, NK cell, B cell),

3. Antibody titers,

4. Thymic production and measurement of T/B lymphocyte repertoire,

5. Measurement of the functions (ELISPOT, intracellular cytokine measurement with flow cytometry tetramerous measurement) methods can be used (15).

In currents studies, some methods showed promising results for the strengthening of immune recovery after HSCT including IL-7, keratinocyte growth factor, growth hormone, T lymphocyte precursor cultures being given to the recipient, and sex hormone ablation techniques (15).

In conclusion, stem cell transplantation has been increasingly used in the treatment of many diseases since the 1950s. The fundamental focus of current researchs includes the management of immune reconstitution and complications related to this treatment method. Post-transplant immune recovery may extend up to 5 years depending on to the stem cell source, immunosuppressive interventions used, complications, and on the cell subgroups which can improve at different times. 
Acknowledgement: I would like to thank Dr. Ömer Doğru, faculty member of Marmara University Department of Pediatric Hematology for his valuable contribution and comments.

Peer-review: Externally peer-reviewed.

Author Contributions: Concept -EKK; Design - EKK; Supervision EKK; Materials - EKK; Data Collection and/or Processing - EKK; Analysis and/or Interpretation - EKK; Literature Review - EKK; Writing -EKK; Critical Review - EKK.

Conflict of Interest: No conflict of interest was declared by the author.

Financial Disclosure: The author declared that this study has received no financial support.

\section{References}

1. Smith FO, Reaman GH, Racadio JM (eds). Hematopoietic Cell Transplantation in Children with Cancer. London: Springer-Verlag Berlin Heidelberg, 2014.

2. Passweg $J R$, Baldomero $H$, Bader $P$, Bonini $C$, Cesaro $S$, Dreger $P$, et al; European Society for Blood and Marrow Transplantation (EBMT). Hematopoietic SCT in Europe 2013: recent trends in the use of alternative donors showing more haploidentical donors but fewer cord blood transplants. Bone Marrow Transplant 2015;50(4):476-82.

3. McFarren A, Pulsipher MA. Pretransplantation: indications and timing. In: Brown VI (ed). Hematopoietic Stem Cell Transplantation for the Pediatric Hematologist/Oncologist. Cham, Switzerland: Springer International Publishing AG, 2018:57-76.

4. Danby $R$, Rocha V. Improving engraftment and immune reconstitution in umbilical cord blood transplantation. Front Immunol 2014;5:68.

5. Martin PS, Li S, Nikiforow S, Alyea EP, Alyea EP $3^{\text {rd }}$, Antin JH, Armand $P_{\text {, }}$ et al. Infused total nucleated cell dose is a better predictor of transplant outcomes than CD34 (+) cell number in reduced-intensity mobilized peripheral blood allogeneic hematopoietic cell transplantation. Haematologica 2016;101(4):499-505.
6. Remberger $M$, Törlén J, Ringdén $O$, Engström $M$, Watz $E$, Uhlin $M$, et al. Effect of total nucleated and CD34+ cell dose on outcome after allogeneic hematopoietic stem cell transplantation. Biol Blood Marrow Transplant 2015;21(5):889-93.

7. Ogonek J, KraljJuric M, Ghimire S, Varanasi PR, Holler E, Greinix H, et almmune reconstitution after allogeneic hematopoietic stem cell transplantation. Front Immunol 2016;7:507.

8. Storek J, Geddes M, Khan F, Huard B, Helg C, Chalandon Y, et al. Reconstitution of the immune system after hematopoietic stem cell transplantation in humans. Semin Immunopathol 2008;30:425-37.

9. Fry TJ, Mackall CL. Immune reconstitution following hematopoietic progenitor cell transplantation: challenges for the future. Bone Marrow Transplant 2005;35(Suppl 1):53-7.

10. Trzonkowski P, Bieniaszewska M, Juścińska J, Dobyszuk A, Krzystyniak A, Marek $N$, et al. First-in-man clinical results of the treatment of patients with graft versus host disease with human exvivo expanded CD4+CD25+ CD127- T regulatory cells. Clin Immunol 2009;133(1):22-6.

11. Li Q, Zhai Z, Xu X, Shen Y, Zhang A, Sun Z, et al. Decrease of CD4+CD25+ regulatory $T$ cells and TGF- $\beta$ at early immune reconstitution is associated to the onset and severity of graft-versus-host disease following allogeneic haematogenesis stem cell transplantation. Leuk Res 2010;34(9):1158-68.

12. Hakki $M$, Riddell $S R$, Storek J, Carter RA, Stevens-Ayers $T$, Sudour $P$, et al. Immune reconstitution to cytomegalovirus after allogeneic hematopoietic stem cell transplantation: impact of host factors, drug therapy, and sub-clinical reactivation. Blood 2003;102(8):3060-7.

13. Williams KM, Gress RE. Immune reconstitution and implications for immunotherapy following haematopoietic stem cell transplantation. Best Pract Res Clin Haematol 2008;21(3):579-96.

14. D'Orsogna L, Wright MP, Krueger RG, McKinnon EJ, Buffery SI, Witt CS, et al. Allogeneic hematopoietic stem cell transplantation recipients have defects of both switched and igm memory $B$ cells. Biol Blood Marrow Transplant 2009;15(7):795-803.

15. van den Brink $M R$, Velardi E, Perales MA. Immune reconstitution following stem cell transplantation. Hematology Am Soc Hematol Educ Program 2015;2015:215-9. 